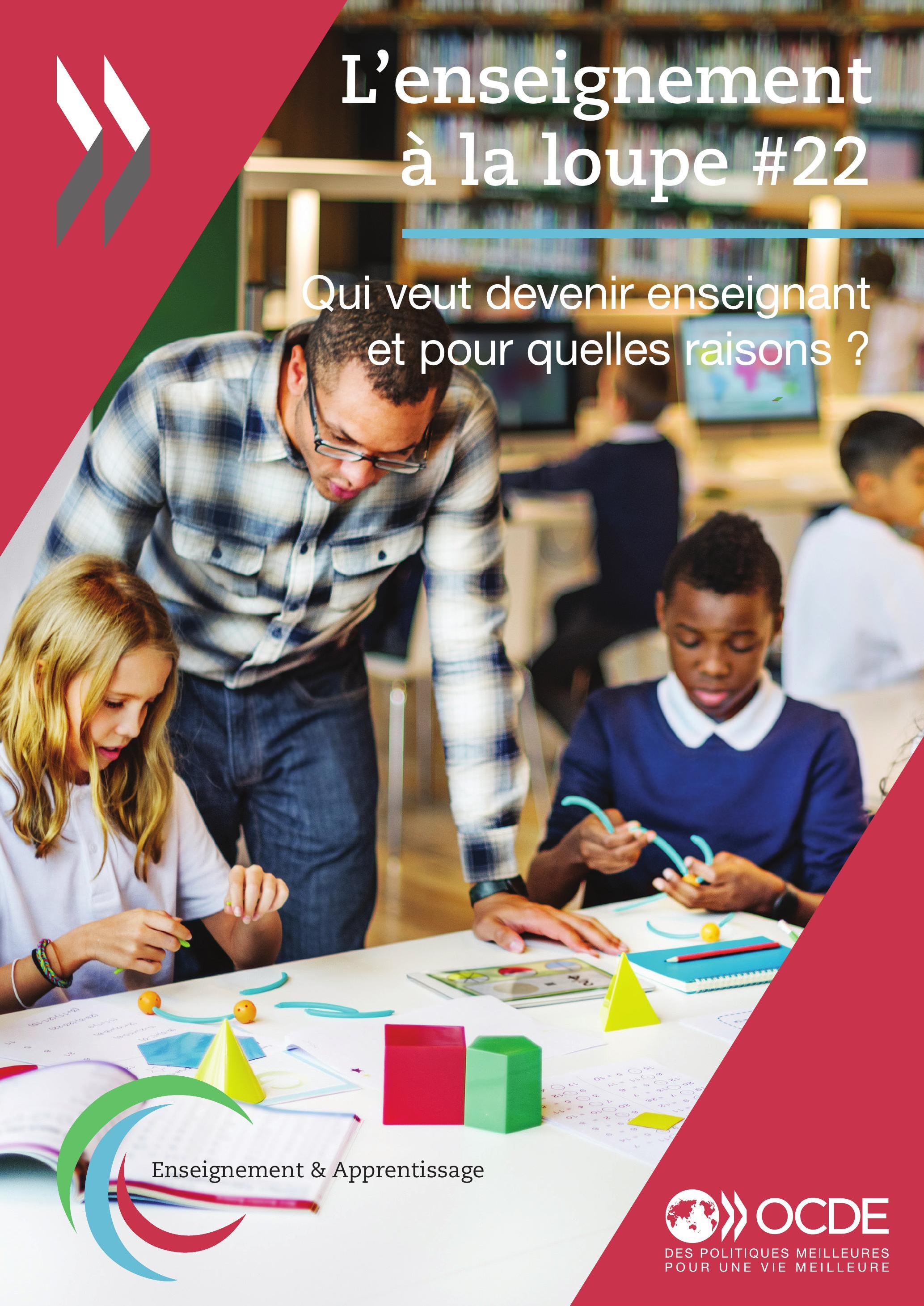




\section{Qui veut devenir enseignant et pour quelles raisons?}

- En moyenne, dans les pays de l'OCDE, 4,2 \% des élèves de 15 ans envisagent une carrière dans l'enseignement - soit un pourcentage supérieur à celui des enseignants dans la population adulte.

- Dans de nombreux pays, les élèves de 15 ans espérant devenir enseignants obtiennent de moins bons scores en mathématiques et en compréhension de l'écrit que ceux envisageant d'exercer d'autres professions nécessitant, à l'instar de l'enseignement, au moins un diplôme universitaire. Toutefois, les données de l'Évaluation des compétences des adultes montrent que les enseignants ont en général des compétences en numératie similaires à celles des autres diplômés universitaires.

- L'écart de compétence entre les élèves espérant devenir enseignants et ceux envisageant d'exercer une autre profession intellectuelle ou scientifique est souvent plus marqué dans les pays peu performants que dans ceux très performants.

- Les pays où le salaire des enseignants est plus élevé (par rapport au PIB) et où la profession d'enseignant jouit d'un meilleur statut social présentent, en moyenne, des pourcentages plus importants d'élèves envisageant de devenir enseignants.

\section{Qui aspire à une carrière dans l'enseignement?}

La prise de conscience croissante du rôle déterminant des compétences des enseignants dans la qualité de l'expérience scolaire suscite de plus en plus de préoccupations chez les responsables politiques quant à la difficulté d'attirer des candidats performants et motivés dans la profession d'enseignant. Ces préoccupations sont d'autant plus vives dans les pays manquant d'enseignants ou connaissant une aggravation de ces pénuries au fil du temps.

Les aspirations professionnelles des élèves indiquent dans quelle mesure les adolescents, en particulier ceux dotés d'un fort potentiel académique, envisagent d'embrasser une carrière dans l'enseignement. Les facteurs façonnant les premières aspirations professionnelles déterminent en grande partie le vivier global de candidats potentiels au métier d'enseignant, même si des parcours alternatifs permettant aux adultes d'accéder à la profession à n'importe quel stade de leur vie peuvent atténuer l'influence de ces facteurs.

Une analyse des données 2015 du Programme international pour le suivi des acquis des élèves (PISA) compare entre les pays la mesure dans laquelle les élèves de 15 ans, les élèves très performants et les élèves présentant un profil atypique envisagent de devenir enseignants. Elle tente en outre d'expliquer les variations observées entre les pays en explorant leur lien avec le statut social, la rémunération et les conditions de travail dont bénéficient les enseignants dans les différents pays.

Cette analyse se fonde sur la question suivante, posée aux élèves de 15 ans dans le cadre des enquêtes PISA 2006 et 2015 : "Quel type de métier espérez-vous exercer quand vous aurez environ 30 ans ? ». Elle cible les élèves qui déclarent envisager de devenir enseignants en général ou plus spécifiquement, enseignants du primaire, du secondaire ou en éducation spécialisée.

En moyenne, dans les pays de l'OCDE, 50 \% des élèves indiquent envisager d'exercer une profession intellectuelle ou scientifique, soit une catégorie regroupant des professions jouissant d'un statut élevé et nécessitant généralement un diplôme universitaire. Parmi ceux-ci, 4,2 \% envisagent de devenir enseignants (graphique 1). Par comparaison, les enseignants du primaire et des premier et deuxième cycles du secondaire représentaient en 2013 environ 2,4 \% de la population active dans les pays de l'OCDE. II en ressort que, dans l'ensemble, le pourcentage d'élèves envisageant de devenir enseignants est supérieur à celui de la population en âge de travailler exerçant aujourd'hui ce métier. À ce stade précoce de l'orientation professionnelle, on ne peut toutefois pas conclure qu'il existe un manque global de candidats à la profession d'enseignant. De fait, l'enseignement jouit, au même titre que les métiers de la santé, d'un avantage net par rapport à d'autres professions : tous les élèves de 15 ans ont connaissance du métier d'enseignant, ont déjà été en contact avec des enseignants et ont au moins pu se faire une idée approximative de ce qu'ils font et des conditions dans lesquelles ils travaillent. 


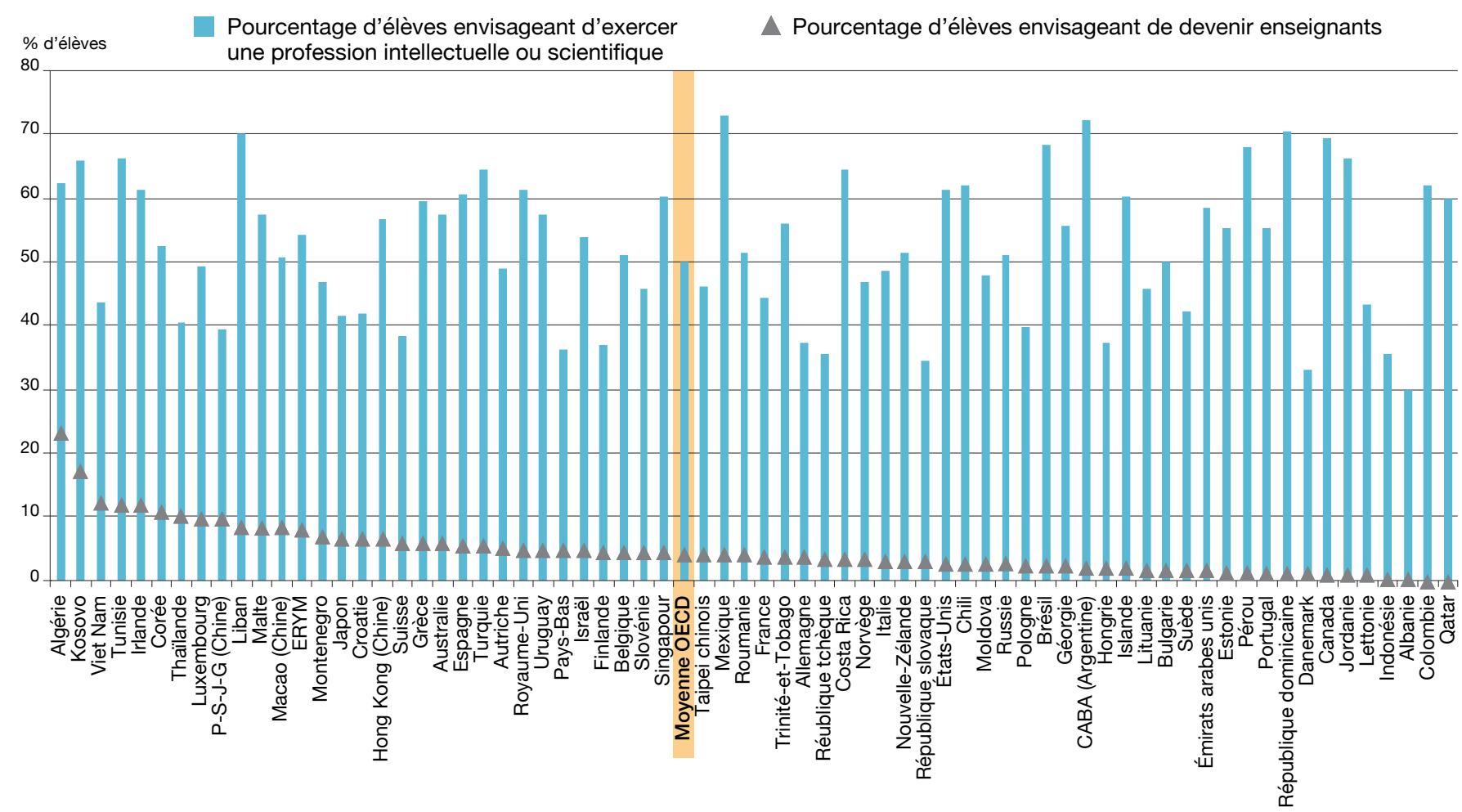

Remarques : Les pays sont classés par ordre décroissant du pourcentage d'élèves de 15 ans envisageant d'exercer le métier d'enseignant quand ils auront 30 ans. Les professions intellectuelles et scientifiques incluent les scientifiques, les ingénieurs, les médecins, les enseignants, et les commerciaux, juristes, spécialistes des sciences sociales et assimilés.

ERYM : Ex-République yougoslave de Macédoine

Source : OCDE (2018), Effective Teacher Policies: Insights from PISA, PISA, http://dx.doi.org/10.1787/9789264301603-en, tableaux 4.1 et 4.2.

Le pourcentage d'élèves envisageant de devenir enseignants varie sensiblement entre les pays. Cette profession semble particulièrement prisée en Algérie, en Corée, en Irlande, au Kosovo, au Luxembourg, à Pékin-ShanghaiJiangsu-Guangdong (Chine), en Thaillande, en Tunisie et au Viet Nam. À l'inverse, elle attire moins de 1,5\% des jeunes de 15 ans en Albanie, au Canada, en Colombie, au Danemark, aux Émirats arabes unis, en Estonie, en Indonésie, en Jordanie, en Lettonie, au Pérou, au Portugal, au Qatar et en République dominicaine.

Graphique 2. Aspiration à devenir enseignant selon les caractéristiques des élèves

Pourcentage d'élèves envisageant de devenir enseignants (moyenne OCDE), selon...

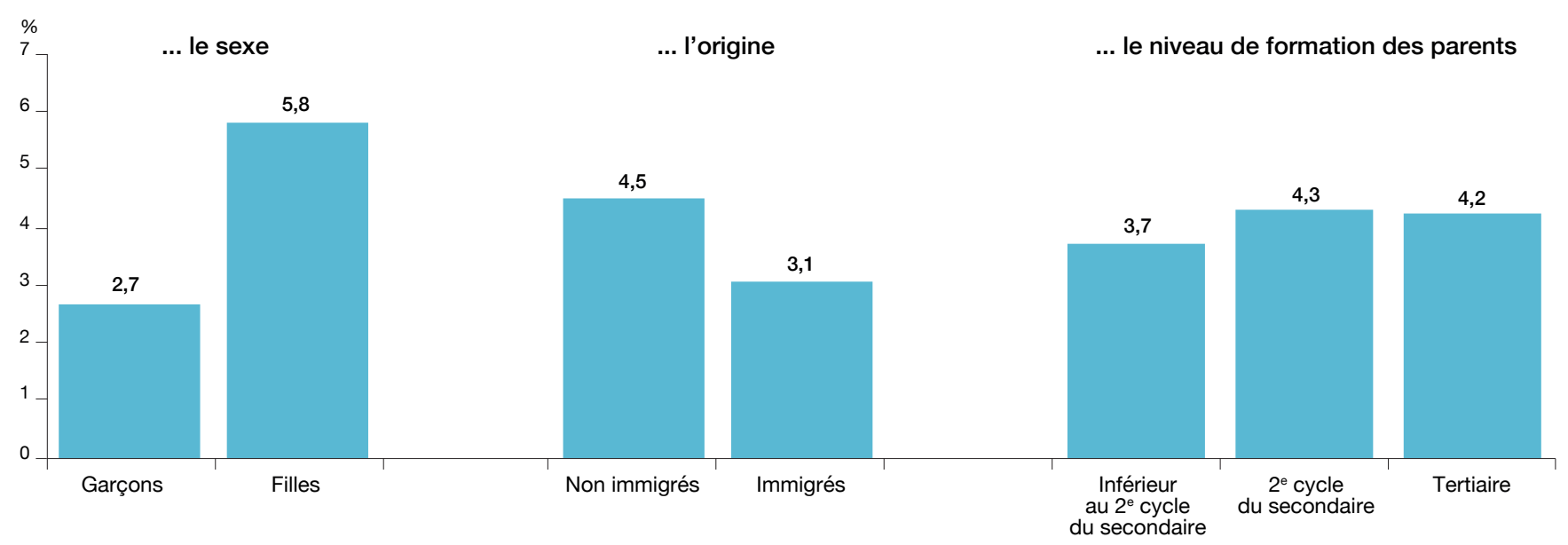

Source : OCDE (2018), Effective Teacher Policies: Insights from PISA, PISA, http://dx.doi.org/10.1787/9789264301603-en, tableaux 4.1 et 4.2. 
Dans la plupart des pays, l'élève type envisageant de devenir enseignant est une fille qui n'est pas issue de l'immigration (graphique 2). En outre, dans nombre de pays, les élèves espérant devenir enseignants obtiennent de moins bons scores aux évaluations PISA de mathématiques et de compréhension de l'écrit que ceux envisageant d'exercer d'autres professions nécessitant, à l'instar de l'enseignement, au moins un diplôme universitaire. Par ailleurs, l'écart de compétence entre les élèves espérant devenir enseignants et ceux envisageant d'exercer un autre type de profession intellectuelle ou scientifique tend à être plus marqué dans les pays peu performants que dans ceux très performants (graphique 3). Ce constat fait écho à des préoccupations de longue date quant à la composition du corps enseignant : dans nombre de pays, les élèves très performants et les hommes sont en effet moins nombreux à choisir de devenir, ou de rester, enseignants. Toutefois, les données de l'Évaluation des compétences des adultes montrent que les enseignants ont en général des compétences en numératie similaires à celles des autres diplômés universitaires.

Graphique 3. Dans quels pays les élèves très performants sont-ils attirés par l'enseignement ?

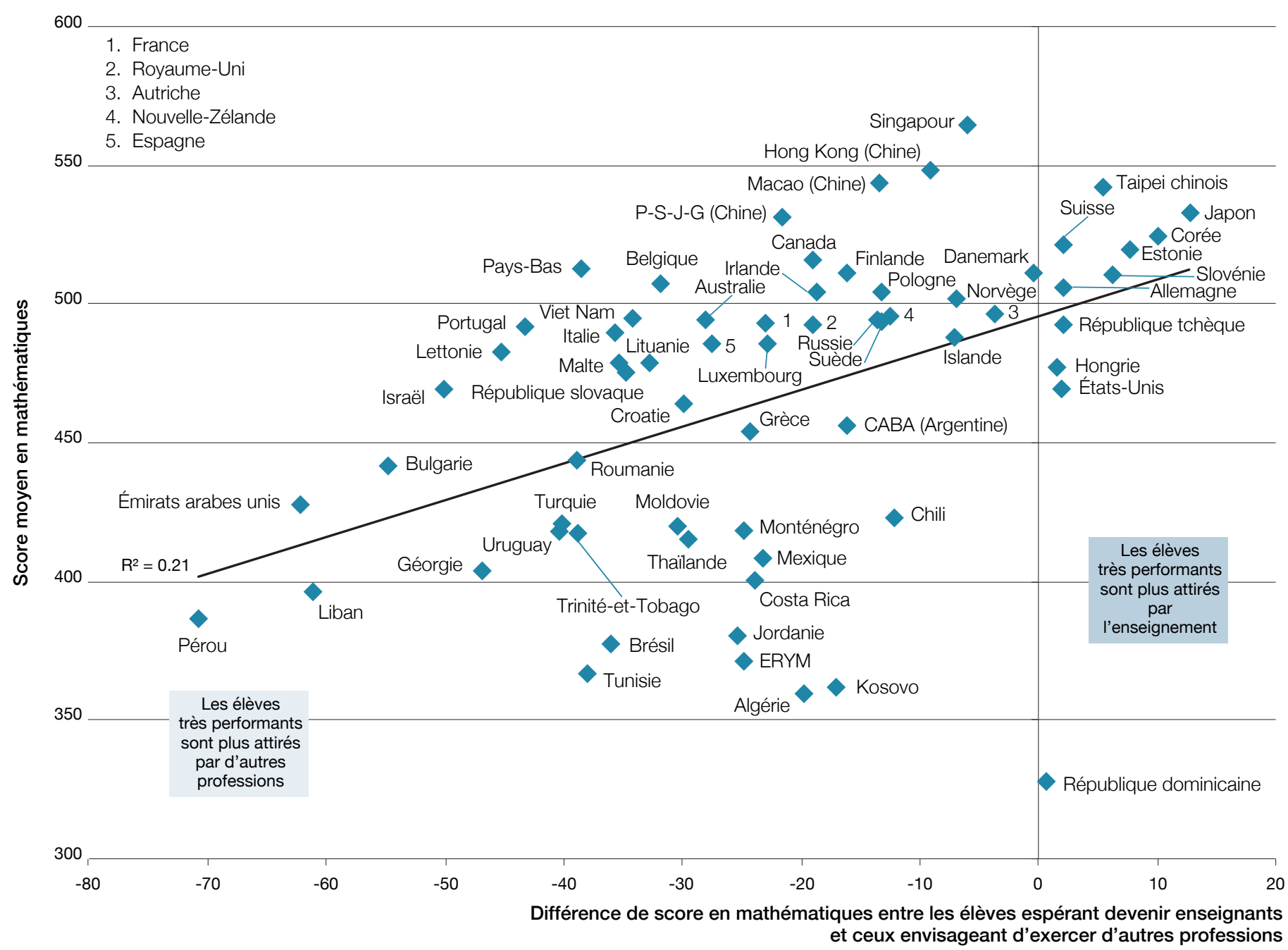

Source : OCDE, Base de données PISA 2015 ; OCDE (2016), Résultats du PISA 2015 (Volume l) : L'excellence et l'équité dans l'éducation, PISA, http://dx.doi.org/10.1787/9789264267534-fr, tableau I.5.3 ; OCDE (2018), Effective Teacher Policies: Insights from PISA, PISA, http://dx.doi.org/10.1787/9789264301603-en, tableau 4.3. 


\section{Comment attirer des candidats plus nombreux et plus qualifiés ?}

Face aux problèmes de pénurie d'enseignants, aux défis du recrutement et aux inquiétudes concernant le statut social de la profession d'enseignant, les responsables politiques doivent trouver comment attirer davantage de candidats dans l'enseignement, en particulier parmi les plus qualifiés. Les enquêtes menées auprès des enseignants en exercice montrent souvent que les enseignants d'aujourd'hui sont fortement motivés par les bénéfices intrinsèques de leur métier - travailler auprès d'enfants, les accompagner dans leur développement et apporter une contribution à la société -, tandis que les études sondant d'importants échantillons de diplômés sur leurs choix de carrière révèlent que le salaire relatif des professions joue un rôle dans leur décision : si le salaire des enseignants était plus élevé, plus d'« enseignants potentiels » envisageraient sérieusement une carrière dans cette profession.

Les données de l'enquête PISA et de l'Enquête internationale sur l'enseignement et l'apprentissage (TALIS) ont été analysées pour explorer le lien entre le pourcentage d'élèves envisageant de devenir enseignants et différentes caractéristiques des élèves et des établissements, ainsi que les différences de rémunération (par rapport au PIB) et de statut social des enseignants entre les pays. Au niveau national, les résultats mettent au jour l'existence d'une association positive entre d'un côté, le salaire et le statut social des enseignants, et de l'autre, les aspirations des élèves à devenir enseignants. La relation entre le salaire et le statut social des enseignants et les aspirations des élèves à devenir enseignants varie selon les compétences scolaires et certaines caractéristiques des élèves, notamment leur sexe, leur statut socio-économique, leur origine et leur performance en mathématiques. Si les résultats indiquent que les garçons sont plus sensibles aux différences salariales, rien ne prouve néanmoins qu'une revalorisation des salaires attirerait davantage les élèves très performants vers le métier d'enseignant que leurs pairs peu performants.

\section{Pour conclure}

II y a davantage d'élèves envisageant de devenir enseignants qu'il n'y a d'enseignants dans la population actuelle. Les systèmes d'éducation pourraient donc déployer davantage de moyens pour encourager et soutenir la poursuite d'une carrière dans l'enseignement parmi tous les élèves qui le désirent. Pour promouvoir l'enseignement comme choix de carrière, en particulier auprès des élèves très performants, la qualité de l'emploi revêt au moins autant d'importance que la rémunération. Différents moyens d'action prometteurs pourraient notamment prévoir de transformer l'organisation de travail des établissements, d'associer les enseignants au processus décisionnel, de renforcer leurs responsabilités de leadership, et de promouvoir l'enseignement comme une profession certes exigeante, mais gratifiante. 
